\title{
Gambogic Acid Inhibits Wnt/ $\beta$-catenin Signaling and Induces ER Stress-Mediated Apoptosis in Human Cholangiocarcinoma
}

\author{
Kanoknetr Suksen ${ }^{1}$, Keatdamrong Janpipatkul ${ }^{1,2}$, Somrudee Reabroi ${ }^{3}$, Natthinee \\ Anantachoke $^{4}$, Vichai Reutrakul ${ }^{5}$, Arthit Chairoungdua ${ }^{1,6,7,8}$, Natthakan \\ Thongon ${ }^{1}$, Kanit Bhukhai ${ }^{\text {* }}$
}

\begin{abstract}
Objective: Gambogic acid (GA) has been reported to induce apoptosis in cholangiocarcinoma (CCA) cell lines. However, the molecular mechanisms underlying its anti-cancer activity remain poorly understood. This study was aimed to investigate GA's effect on human CCA cell lines, KKU-M213 and HuCCA-1, and its associated mechanisms on Wnt/ $\beta$-catenin signaling pathway. Methods: Cell viability, apoptosis, and cell cycle analysis were conducted by MTT and flow cytometry. The effect of GA mediated Wnt/ $\beta$-catenin and ER stress were determined by luciferase-reporter assay, qRT-PCR, and western blot analysis. Results: GA exhibited potent cytotoxicity in CCA cells which was associated with significantly inhibited cell proliferation, promoted G1 arrest, and activated caspase 3 mediated-apoptosis. GA attenuated $\beta$-catenin transcriptional levels, decreased $\beta$-catenin protein, and suppressed the expression of $c-M y c$, a downstream target gene of Wnt/ $\beta$-catenin signaling. GA activated genes involved in ER stress mechanism in KKU-M213 and enhanced CCA's sensitivity to gemcitabine. Conclusion: Our findings reveal that the molecular mechanism underpinning anti-cancer effect of GA is partially mediated through the inhibition of Wnt/ $\beta$-catenin signaling pathway and induction of ER stress induced-apoptosis. GA may serve as a promising therapeutic modality for amelioration of gemcitabine-induced toxicity in CCA.
\end{abstract}

Keywords: Gambogic acid- cholangiocarcinoma- anti-cancer- Wnt/ $\beta$-catenin signaling pathway- ER stress

Asian Pac J Cancer Prev, 22 (6), 1913-1920

\section{Introduction}

Cholangiocarcinoma (CCA) is the second most common primary liver tumor with a morphological feature of the aggressive malignant proliferation of biliary duct epithelial cells. While CCA incidence rates are increasing globally, the highest incidence and mortality rates are in the North-eastern Thailand specifically in the endemic area of liver fluke infestations (Banales et al., 2016; Prakobwong et al., 2017; Alsaleh et al., 2019; Chansitthichok et al., 2020). Indeed, surgery is the principal treatment that provided the best survival prognosis of CCA patients (Chanchai et al., 2019), however, $40-85 \%$ of all patients have recurrent disease even after tumor excision (Vogel et al., 2014; Marin et al., 2018) and surgical resection has become an incurable option for most CCA patients in advanced stages of the disease (Yao et al., 2014). Due to rapid progression of CCA, therapeutic effort has been directed to identifying and modulating key molecular pathways relevant to cancer growth. Chemotherapy for advanced CCA is largely ineffective due to the emerging of resistance in CCA (Sawasdee et al., 2020). Therefore, targeting essential signaling pathways mediated CCA progression is critical for understanding and developing treatments.

Wnt/ $\beta$-catenin signaling pathway plays important roles in normal tissue development, including cell proliferation and differentiation (Nusse and Clevers, 2017). Cytosolic $\beta$-catenin protein is a principal mediator of $\mathrm{Wnt} / \beta$-catenin signaling and its phosphorylation, ubiquitination, and degradation are governed by a destruction complex comprising of Axin, adenomatous polyposis coli (APC),

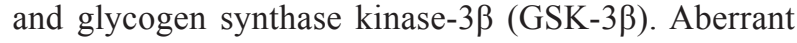
activation of this complex gives rise to the accumulation

${ }^{1}$ Department of Physiology, Faculty of Science, Mahidol University, Bangkok, Thailand. ${ }^{2}$ Department of Basic Medical Science, Faculty of Medicine Vajira Hospital, Navamindradhiraj University, Bangkok, Thailand. ${ }^{3}$ Department of Pharmacology, Faculty of Science, Mahidol University, Bangkok, Thailand. ${ }^{4}$ Department of Pharmacognosy, Faculty of Pharmacy, Mahidol University, Bangkok, Thailand. ${ }^{5}$ Department of Chemistry and Center of Excellence for Innovation in Chemistry (PERCH-CIC), Faculty of Science, Mahidol University, Bangkok, Thailand. ${ }^{6}$ Excellent Center for Drug Discovery (ECDD), Mahidol University, Bangkok, Thailand. ${ }^{7}$ Toxicology Graduate Program, Faculty of Science, Mahidol University, Bangkok, Thailand. ${ }^{8}$ Center of Excellence on Environmental Health and Toxicology (EHT), Faculty of Science, Mahidol University, Bangkok, Thailand. *For Correspondence: kanit.bhu@mahidol.ac.th 
of $\beta$-catenin in nucleus which functions as a transcriptional coactivator of transcription factors included in the T-cell factor and lymphoid enhancer factor (TCF/LEF) family and promotes the transcription of Wnt responsive genes including $c-M y c$ and $C C N D 1$. Hyper-activation of $\mathrm{Wnt} / \beta$-catenin signaling observe in several types of human cancers which drives tumor initiation and progression (Tripathy et al., 2018). Thus, the discovery of compounds targeting $\mathrm{Wnt} / \beta$-catenin signaling has potential as a therapeutic strategy for CCA.

Xanthone is isolated from the resin of Garcinia hanburyi (Reutrakul et al., 2007; Hahnvajanawong et al., 2010). Among the naturally occurring caged xanthones, Gambogic acid (GA) has been identified as an effective anti-cancer agent in a variety of tumors (Zhang et al., 2004; Kashyap et al., 2016). GA has a unique structure in which the planar structure of xanthone (Figure 1A) is recognized as an efficient DNA intercalator possessing anti-cancer activities (Qin et al., 2007) and an intact ABC ring of caged xanthone compounds reveals an optimisation of a given bioactivity against cancers. In particular, the 9,10 carbon-carbon double bond of the $\alpha, \beta$-unsaturated ketone is essential for their cytotoxic and apoptotic activities (Zhang et al., 2004). Accumulating evidence indicates that GA strongly induces ER stress, suppresses human growth factor receptors (He et al., 2013), and regulates several signaling pathways which are crucial for its molecular processes in cancers. However, the specific molecular mechanisms related to anti-cancer activity of GA in CCA cells are still unclear. Here we aimed to investigate the effect of GA on the modulation of Wnt/ $\beta$-catenin signaling pathway in CCA malignancy phenotypes.

\section{Materials and Methods}

\section{Chemicals, plasmids, and reagents}

Gambogic acid (GA) (Figure 1A) was isolated from Garcinia hanburyi (Family Guttiferae) as previously described (Reutrakul et al., 2007). Dual-luciferase reporter assay was purchased from Promega (Madison, WI). $\beta$-catenin-pcDNA3.1(+), TOPflash, FOPflash, S33Y, and Renilla were previously described elsewhere (Chairoungdua et al., 2010). The following reagents were used: MTT (3-(4, 5-dimethylthiazol-2-yl)-2, 5-diphenyl tetrasolium bromide) (Sigma-Aldrich, St. Louis, MO) and cell Proliferation ELISA and BrdU colorimetric assay were from Roche (Roche, Mannheim, Germany).

\section{Cell culture}

KKU-M213 cells were purchased from JCRB (Osaka, Japan). HEK 293 T cells were obtained from American Type Culture Collection (ATCC, Manassas, VA). HuCCA-1 cell line was kindly provided by Dr. Stitaya Sirisinha, Department of Microbiology, Faculty of Science, Mahidol University. KKU-M213 and HuCCA-1 cells were cultured in Ham's F-12 medium (Invitrogen, Carlsbad, CA). HEK 293 T cells were maintained in Minimum Essential Medium (MEM). All culture mediums were supplemented with $10 \%$ Fetal bovine serum (FBS) and $100 \mathrm{U} / \mathrm{ml}$ penicillin/streptomycin and cells were cultured at $37^{\circ} \mathrm{C}$ in a humidified atmosphere containing
$5 \% \mathrm{CO}_{2}$ incubator.

\section{Cell proliferation and cytotoxic assay}

Cell proliferation was determined by a Cell Proliferation ELISA and BrdU colorimetric assay according to the manufacturer's instructions. GA treated-cells were incubated in BrdU labelling solution for $2 \mathrm{~h}$ at $37^{\circ} \mathrm{C}$ and BrdU-POD antibody was added and incubated for $90 \mathrm{~min}$ at room temperature (RT). Cell proliferation was determined by measuring the absorbance at $450 \mathrm{~nm}$ using a Microplate Spectrophotometer. Cell viability was determined by MTT assay. Briefly, cells were incubated with $0.5 \%$ MTT reagent for $4 \mathrm{~h}$ at $37^{\circ} \mathrm{C}$ to allow the formation of a violet formazan precipitate. Then, supernatant was removed and formazan crystals were dissolved in 100\% DMSO prior to measuring absorbance at $540 \mathrm{~nm}$ by a Multiskan GO Microplate Spectrophotometer (Thermo Scientific, Waltham, MA).

\section{Annexin V and Propidium iodide (PI) staining}

Cells were treated with GA for $24 \mathrm{~h}$. They were harvested and incubated with Annexin V-FITC and PI for 15 min at RT in the dark. The frequency of apoptotic cells was determined by BD FACSCanto ${ }^{\mathrm{TM}}$ flow cytometer and analyzed with BD FACSDiva software (BD Bioscience, San Jose, CA).

\section{Cell cycle analysis}

CCA cells were treated with GA for $24 \mathrm{~h}$, fixed with cold $70 \%$ ethanol overnight at $20^{\circ} \mathrm{C}$ and stained in $\mathrm{PI} /$ RNase Staining Buffer in the dark for $30 \mathrm{~min}$ at $37^{\circ} \mathrm{C}$. Cell cycle analysis was performed by BD FACSCanto ${ }^{\mathrm{TM}}$ flow cytometer and analysed with a BD FACSDiva software.

\section{Luciferase reporter assay}

HEK 293 T cells were transiently transfected with FLAG- $\beta$-catenin, TOPflash/TCF reporter, FOPflash, and Renilla luciferase reporter plasmids using lipofectamine 3000 according to the manufacturer's instructions (Invitrogen, Carlsbad, CA). Twenty-four hours after transfection, cells were treated with GA for $24 \mathrm{~h}$ and subjected to reporter assay analysis using Dual-luciferase reporter kit.

\section{Western blot analysis}

Total protein lysates were harvested using a modified lysis buffer as previously described (Bhukhai et al., 2012). Samples were resolved by $10 \%$ of sodium dodecyl sulfate polyacrylamide gel electrophoresis (SDS-PAGE). The following antibodies were used: $\beta$-catenin (H-102) (Santa Cruz Biotechnology, CA), $\beta$-actin (SigmaAldrich), Cleaved-caspase 3, p-GSK3 (Ser 9), and gH2AX (Cell signaling, MA). Membranes were then probed with HRP-conjugated secondary antibodies for $1 \mathrm{~h}$. The signal was detected using SuperSignal West Pico Chemiluminescent ECL system (Thermo Scientific, Waltham, MA).

\section{$R N A$ extraction and real time $P C R$}

Total RNAs were isolated using Trizol reagent according to the manufacturer's instructions. The 
quantity of total RNA was determined by NanoDrop 2000 (Thermo Scientific, Waltham, MA). First-strand complementary DNA (cDNA) synthesis was performed using iScriptTM cDNA synthesis kit (Bio-Rad, Hercules, CA). For real-time quantitative PCR, cDNA samples were amplified using SYBR Green I dye and conducted with ABI PRISM7500 Sequence Detection System and analysis software (Applied Biosystem; Bedford, MA). The mRNA expression was normalized to the levels of endogenous GAPDH and sequences of primers are GRP78 (FW: CCCGAGAACACGGTCTTTGA, RW: TCAACCACCTTGAACGGCAA), IRE-1 (FW: CGGCCTCGGGATTTTTGGAA, RW: TTGAGCCTGTCCTCTTGCTG), XBP-1 (FW: GGAAGCCAAGGGGAATGAAGIRWOCTGCAGAGGTGCACGTAG), CHOP (FW: GGAACCTGAGGAGAGAGTGTTC, RW: TGCCATCTCTGCAGTTGGAT), and GAPDH (FW: ATGCCCCCATGT TCGTCATG, RW : GCAGGAGGCATTGCTGAT).

\section{Statistical analysis}

Data are represented as mean and standard deviation (mean $\pm \mathrm{SD}$ ) or mean \pm standard error of mean (SEM) for IC50 (Supplementary Table 1). Data were analysed using the statistical software package, GraphPad Prism version 8.2 (GraphPad, CA, USA). The statistical differences among groups were analysed by a one-way ANOVA followed by Tukey-Kramer post-hoc test and paired t-test was used when applicable. Each value is represented as means \pm SD of three independent experiments. Statistical significance was considered when values of $*, p<0.05$, $* *, \mathrm{p}<0.01, * * *, \mathrm{p}<0.001$ compared with vehicle control.

\section{Results}

GA exhibits cytotoxic and anti-proliferative effects in CCA cells

To investigate the effects of GA in CCA, KKU-M213 and HuCCA-1 cells were treated with various concentrations of GA for 24-72 $\mathrm{h}$ and cell viability was determined by MTT assay. Exposure of GA markedly reduced cell viability in time and dose-dependent manners in both CCA cell lines. The IC50 values of GA in KKU-M213 and HuCCA-1 cells are shown in Supplementary Table 1 . These results aligned with a significantly decreased cell proliferation assessed by a BrdU assay (Figures $1 \mathrm{~B}$ and 1C). GA significantly inhibited KKU-M213 and HuCCA-1 cell proliferation ( $p$ $<0.01$ ) suggesting the potential role of GA in inhibiting cell viability and proliferation of CCAs.

GA induces cell cycle arrest and apoptosis in CCA cells

To demonstrate the effect of GA on cell cycle distribution, GA-treated CCA cells were subjected to flow cytometry analysis. As shown in Figure 1D, GA increased the accumulation of KKU-M213 cells at sub G1 phase in a dose-dependent manner. The frequency of sub G1 cells was increased from $1.9 \%$ in the vehicle control to $4.7 \%, 9.3 \%$, and $27.9 \%$ by treatments of GA at $0.5,1.0$, and $2.5 \mu \mathrm{M}$, respectively. In contrast, the effect of GA on cell cycle arrest was less significant in HuCCA-1 cells than KKU-M213. We further investigated whether GA induces apoptotic cell death in CCA cells. Flow cytometry analysis of GA-treated CCA cells doubly labelled with annexin $\mathrm{V}$ and PI revealed that the frequency of annexin $\mathrm{V}$ positive cells was significantly increased in KKU-M213 cells by GA (Figure 1E). Despite GA exhibited cytotoxicity and anti-proliferative effects in HuCCA-1 cells, but GA (at 0.5-2.5 $\mu \mathrm{M}$ ) failed to induce apoptosis and cell cycle arrest in HuCCA-1 cells (Figure S1A) suggesting that the sensitivity to GA might be due to a cell line-dependence. In addition, we found that GA induced the activation of $\mathrm{gH} 2 \mathrm{AX}$, a biomarker of DNA double-strand breaks (Figure 1F). This finding accompanied with an increase in the number of cells with nuclear fragmentation and chromatin condensation, a hallmark of apoptosis GA-treated CCA cells (Figure S1B) indicating the potential role of GA inducing DNA damage-mediated cell death. Consistently, GA-induced apoptosis was confirmed by increased cleaved-caspase 3 in KKU-M213 cells (Figure 1F). These results strongly suggest that GA induces DNA damage activation and possesses its anti-cancer effect through the induction of caspase 3-dependent apoptosis in KKU-M213 cells.

\section{GA inhibits Wnt/ $\beta$-catenin signaling}

$\mathrm{Wnt} / \beta$-catenin signaling pathway is activated in multiple cancers (Tripathy et al., 2018) and caspase 3-dependent apoptosis has been reported to be partially mediated through $\mathrm{Wnt} / \beta$-catenin signaling pathway (Wu et al., 2014). To address the probable anti-cancer mechanism of GA on Wnt/ $\beta$-catenin signaling pathway, we examined the effect of GA mediated-Wnt $/ \beta$-catenin transcriptional activity using a luciferase reporter assay in HEK 293 cells which is lack of endogenous levels of $\beta$-catenin. Cells were transiently transfected with TOPflash, FLAG- $\beta$-catenin, and Renilla plasmids, and then treated with GA. We observed that $\mathrm{TCF} / \beta$-catenin transcriptional activity was significantly inhibited by GA in a dose-dependent manner (Figure 2A). The inhibitory effect of GA on $\beta$-catenin dependent signaling was further confirmed by using FOPflash, an LEF mutant reporter plasmid, by which the expression of $\beta$-catenin failed to induce $\mathrm{Wnt} / \beta$-catenin transcriptional activity. As expected, GA did not inhibit Wnt/ $\beta$-catenin activity in FOPflash transfected HEK 293T cells (Figure 2B) indicating the modulation of GA on this pathway is $\beta$-catenin dependent-TCF activation. We next performed the TCF/LEF-luciferase reporter assay in KKU-M213 and HuCCA-1 cells. A sublethal concentration of GA at $1 \mu \mathrm{M}$ was administrated to the cells and we observed that GA at $1 \mu \mathrm{M}$ significantly attenuated reporter activity in KKU-M213 (Figure 2C) as well as the trend of reduction of reporter activity was noticed in HuCCA-1 cells (Figure $\mathrm{S} 1 \mathrm{C})$. We next examined the level of $\beta$-catenin protein, which is a key component in $\mathrm{Wnt} / \beta$-catenin signaling pathway. GA slightly attenuated $\beta$-catenin protein levels in KKU-M213 (Figure 2D). These results suggested the inhibitory effect of GA on $\mathrm{Wnt} / \beta$-catenin signaling in KKU-M213.

$\beta$-catenin phosphorylation is mediated by GSK-3 $\beta$ and Asian Pacific Journal of Cancer Prevention, Vol 221915 
A

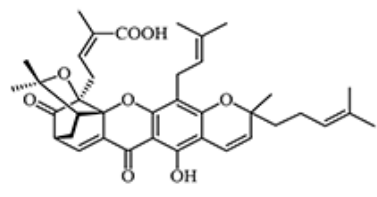

Gambogic acid (GA)
B

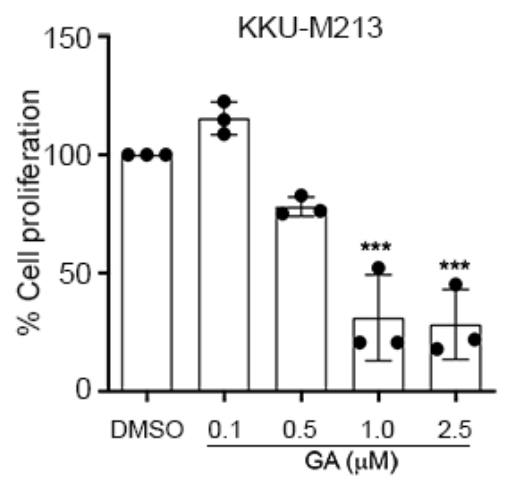

C

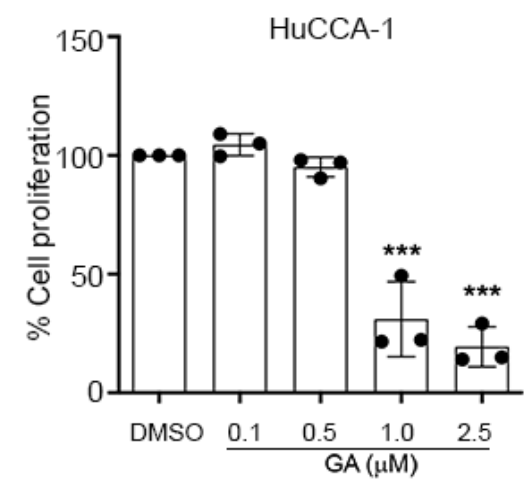

D
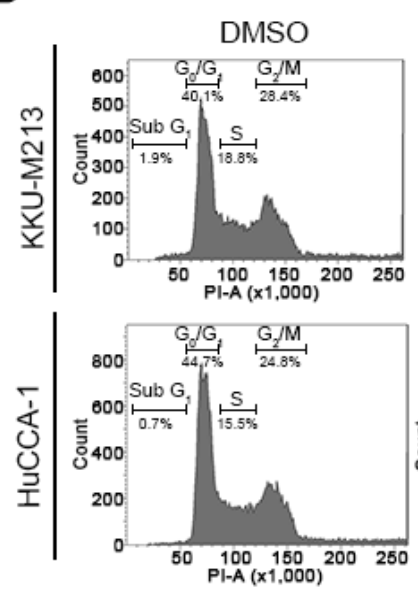

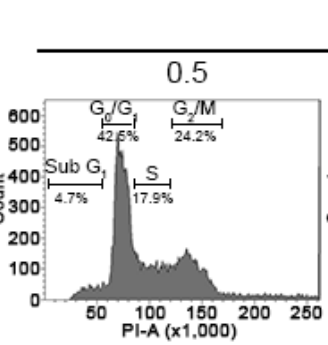

$\mathrm{GA}(\mu \mathrm{M})$
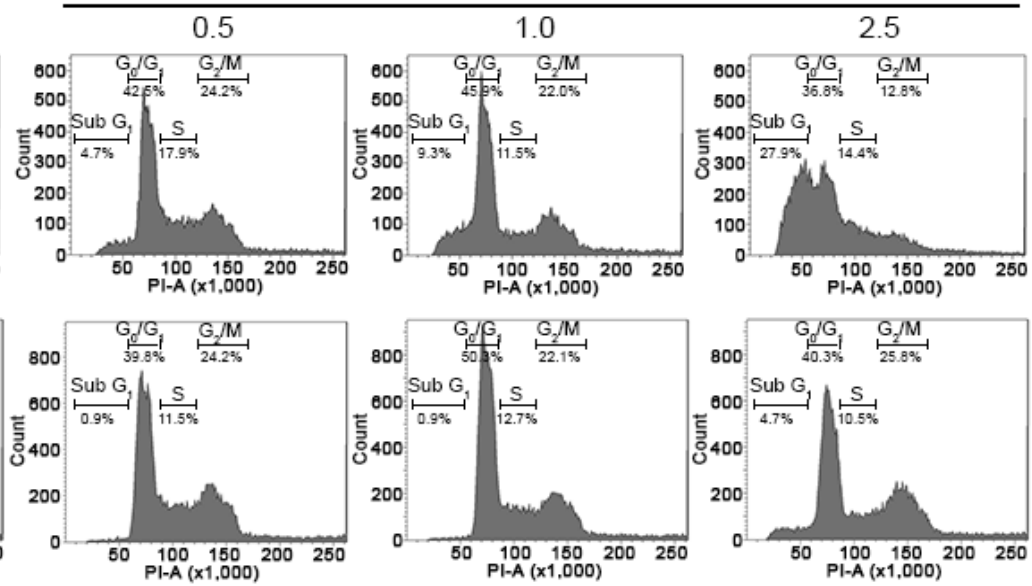

E

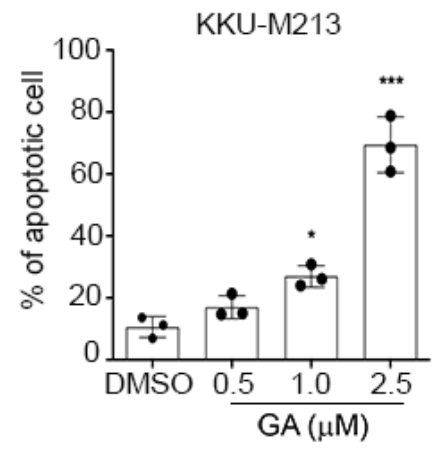

F

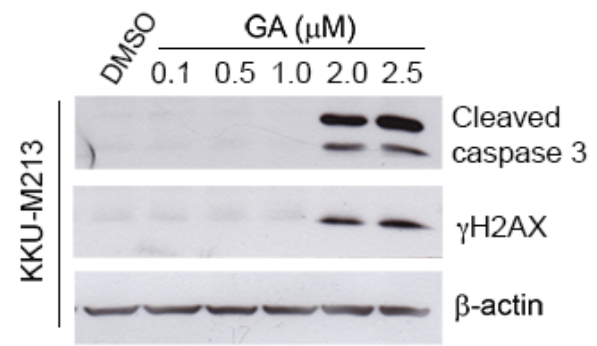

Figure 1. GA Inhibits Cell Proliferation and Induces Apoptosis in CCA. (A) Chemical structures of Gambogic acid (GA) (B-C) The anti-proliferative effect of GA in KKU-M213 and HuCCA-1 cells evaluated by BrdU cell proliferation assay $24 \mathrm{~h}$ after treatment. (D) Cell cycle progression of CCA cells treated with GA for $24 \mathrm{~h}$. (E) The percentage of apoptotic cells in GA treated-KKU-M213 at 24 h. (F) Representative Western blot of cleaved-caspase 3 and $\gamma \mathrm{H} 2 \mathrm{AX}$ of GA treated-KKU-M213 for $24 \mathrm{~h}$ is shown. $\beta$-actin was used as a loading control.

it results in targeting for degradation by the proteasome pathway (Nusse and Clevers, 2017). Mechanistically, to address whether GA attenuated-Wnt/ $\beta$-catenin signaling is mediated through GSK-3 $\beta$, we evaluated the effect of GA on Wnt/ $\beta$-catenin signaling using a constitutively active $\beta$-catenin mutant, S33Y in HEK 293T cells. S33Y is insensitive to GSK-3 $\beta$-mediated phosphorylation, thereby blocking proteasome-induced protein degradation. We observed that the sublethal concentration of GA at $1 \mu \mathrm{M}$ significantly decreased $\mathrm{Wnt} / \beta$-catenin activity in HEK
293 T cells overexpressing wild-type $\beta$-catenin (Figure S2A) but did not affected in S33Y- $\beta$-catenin mutant. These results were confirmed by co-treatment with $\mathrm{LiCl}$, a known GSK-3 $\beta$ inhibitor and we observed that $\mathrm{LiCl}$ treatment resulted in greater activation of TOPflash luciferase activity. However, GA failed to inhibit Wnt/ $\beta$-catenin signaling in the presence of $\mathrm{LiCl}$ in HEK $293 \mathrm{~T}$ cells (Figure S2B) suggesting that GA inhibited Wnt/ $\beta$-catenin signaling is a GSK-3 $\beta$ dependence activation in HEK 293 T cells. We further determined the effect of GA on 
A

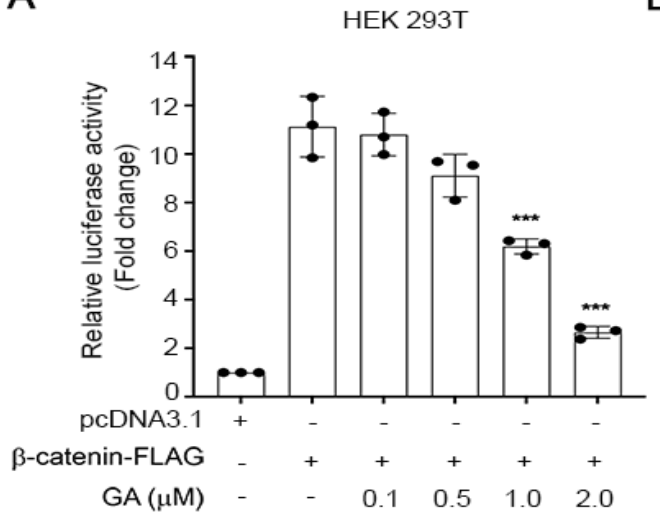

C

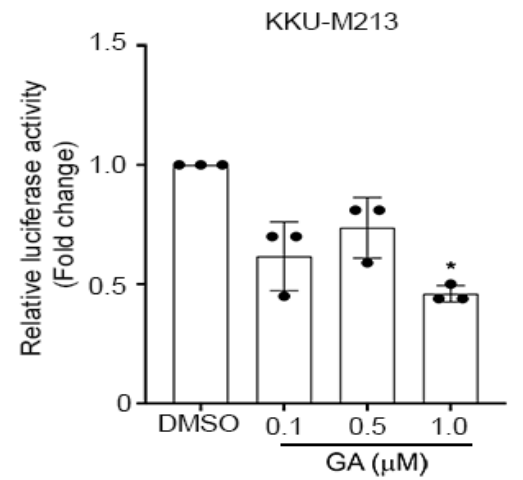

B

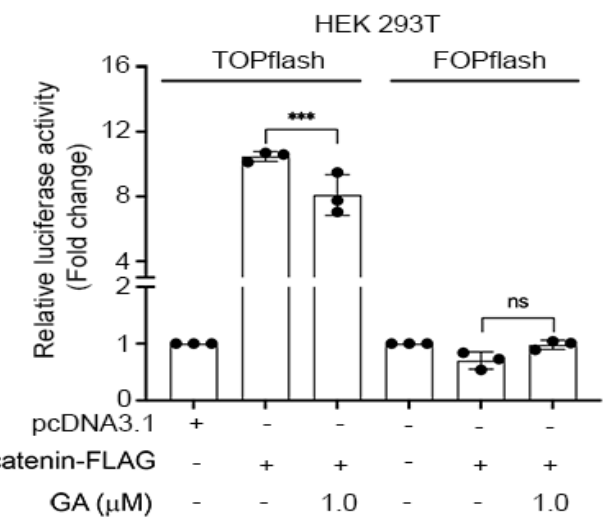

D

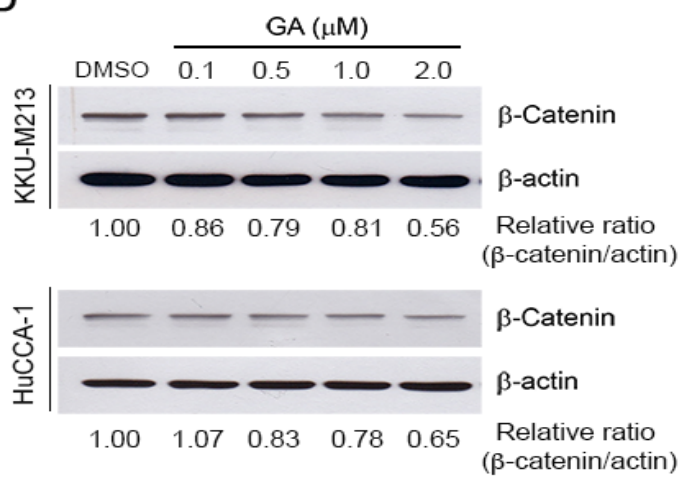

Figure 2. GA Attenuates TCF/LEF Reporter Activity and $\beta$-catenin Protein Expression. TCF/LEF reporter activity of GA treated HEK 293T cells over expressing TOPflash (A), TOPflash or FOPflash (B) for 24h. (C) TCF/LEF luciferase activity of GA treated KKU-M213 cells. Relative luciferase activity was quantified by measuring the relative firefly luciferase activity units and normalized to Renilla luciferase activity. (D) Representative Western blot of $\beta$-catenin in GA treated KKU-M213 and HuCCA-1 for $24 \mathrm{~h}$.

A

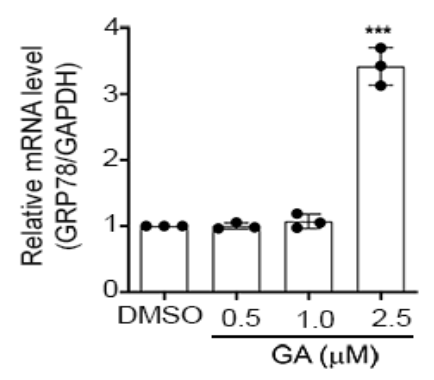

D

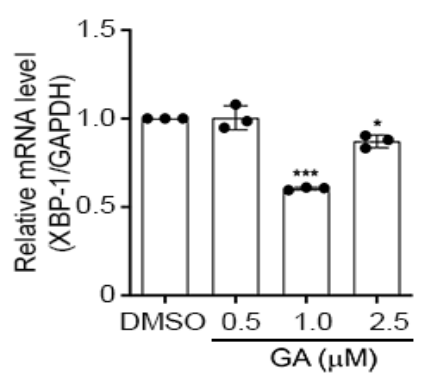

B

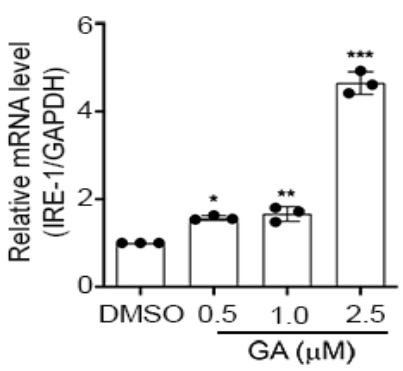

E

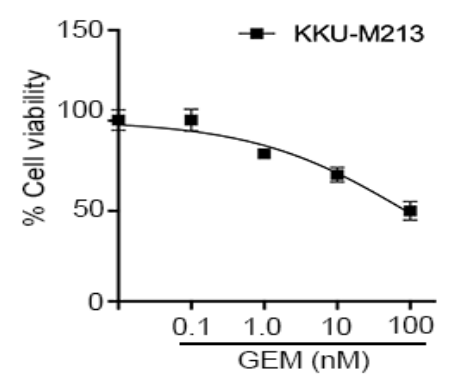

C

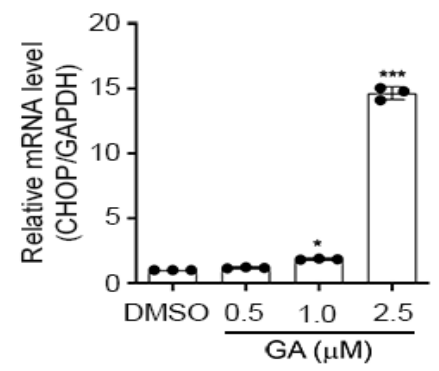

$\mathrm{F}$

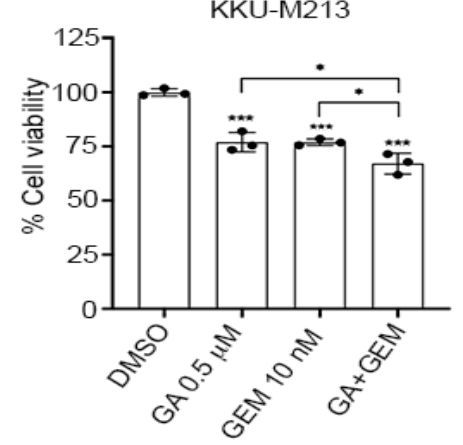

Figure 3. GA Induces Endoplasmic Reticulum (ER) Stress in CCA Cells. KKU-M213 cells were treated with GA for $24 \mathrm{~h}$ and quantitative RT-PCR for ER stress target genes, GRP78/BiP (A), IRE $1 \alpha(\mathrm{B}), C H O P(\mathrm{C})$, and $X B P 1$ (D) were performed. Data are expressed as fold change compared with those treated with vehicle control and represented as means $\pm \mathrm{SD}(\mathrm{n}=3)$. GAPDH was used as a housekeeping gene. (E) Cell viability of GEM treated KKU-M213 for $72 \mathrm{~h}$. (F) Cell viability experiment of KKU-M213 treated with GA $(0.5 \mu \mathrm{M})$ alone or in combination with GEM (10 $\mathrm{nM}$ ) for $72 \mathrm{~h}$. *, $\mathrm{p}<0.05$ compared to indicated treatments, ${ }^{* *}, \mathrm{p}<0.001$ compared with vehicle control (ANOVA). 


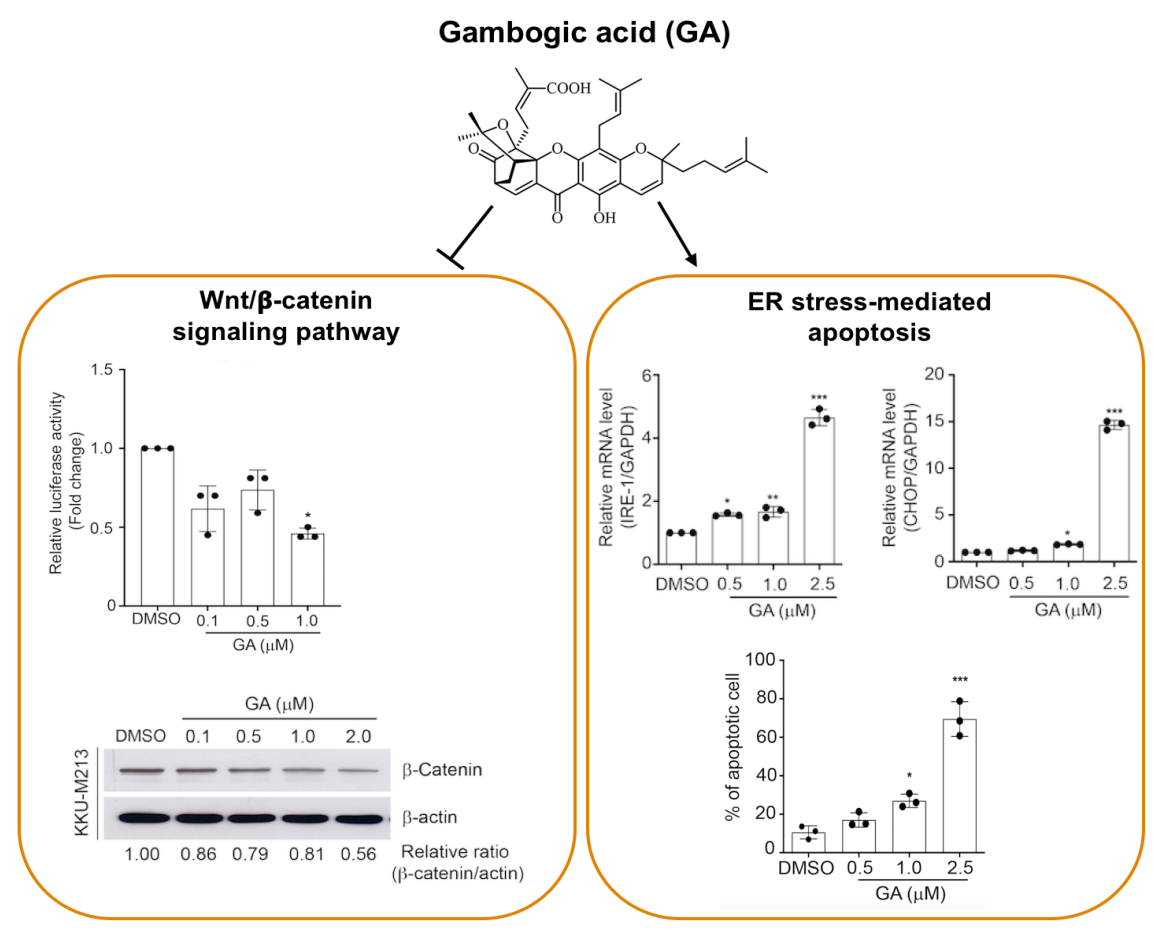

Graph 1. Graphical Abstract

phosphorylation GSK-3 $\beta$ (Ser 9) protein in KKU-M213. In the contrary to HEK 293T, exposure to GA did not alter the levels of $p-G S K-3 \beta$ in KKU-M213 (Figure S2C). This result implies that inhibition of $\mathrm{Wnt} / \beta$-catenin signaling by GA in KKU-M213 cells might be independent from GSK-3 $\beta$ 's action. We speculated that the modulation of GA on the GSK-3 $\beta$ is a cell line specific. However, the pathophysiological consequences of $\mathrm{Wnt} / \beta$-catenin signaling mainly depend on the expression of downstream target genes (Wang et al., 2015). To address this significance, we therefore investigated the effect of GA on the expression of downstream Wnt target genes including AXIN2 and $c-M y c$. GA induced a dose-dependent activation of $A X I N 2$, a negative feedback regulator of Wnt/ $\beta$-catenin signaling (Figure S2D), whereas markedly suppressed $c-M y c$ mRNA expression (Figure S2E). These results indicate that GA potentially inhibited the TCF/ LEF-dependent activation of the canonical Wnt/ $\beta$-catenin signaling and its downstream target gene $c-M y c$.

GA induces endoplasmic reticulum (ER) stress in CCA cells

Sustained ER stress results in cancer cell death and impairs Wnt protein processing (Verras et al., 2008). ER stress induced-apoptotic cell death $\mathrm{h}$ as been reported in CCA (Vaeteewoottacharn et al., 2013). To determine whether GA induces ER stress promoted-apoptosis cell death in KKU-M 213cells, the mRNA levels of critical genes involved in ER stress mechanism including GRP78/ BiP, IRE $1 \alpha, X B P 1$, and CHOP were determined. GRP78/ $B i P, I R E 1 \alpha$, and $C H O P$ mRNAs were significantly up-regulated in KKU-M213 cells exposed to GA for 24 $\mathrm{h}$, with a maximal functional response at $2.5 \mu \mathrm{M}$ (Figure $3 \mathrm{~A}-3 \mathrm{C}$ ) in accordance with our previous experiment showing that caspase 3 -dependent apoptosis was induced by GA. It should be noted that the XBP1 mRNA was significantly decreased by $1-2.5 \mu \mathrm{M}$ of GA treatment (Figure 3D). These results demonstrate that GA induced ER stress-mediated apoptosis in KKU-M213 cells.

GA enhances the sensitivity of CCA cells to Gemcitabine Gemcitabine (GEM) has been used as an established regimen for advanced biliary cancer (Abdel-Rahman et al., 2018). GEM decreased cell viability of KKU-M213 in a dose-dependent manner (Figure 3E). To evaluate whether GA efficiently enhances CCA cell's sensitivity to GEM, KKU-M213 cells were treated with GA alone or in combination with GEM for $72 \mathrm{~h}$. We observed that GA sensitized GEM inhibition CCA cell viability (Figure 3F). These results highlight the possibility that the combination treatment of GA and GEM can potentially improve CAA treatment.

\section{Discussion}

In this present study, we demonstrated that the anti-cancer effects of GA against KKU-M213 partially mediates through the inhibition of $\mathrm{Wnt} / \beta$-catenin signaling pathway. GA potently inhibited CCA cell proliferation, arrested cell cycle, and substantially induced ER stress and apoptosis which significantly impacted on CCA cell survival. To our knowledge, this is the first report showing that the inhibitory effect of GA on $\mathrm{Wnt} / \beta$-catenin signaling pathway and its activation to induce ER stress mediated-apoptosis may cooperate to suppress the progression of CCA cells.

We observed potent cytotoxic effect of GA in both KKU-M213 and HuCCA-1 cell lines. Despite GA exhibited cytotoxicity and anti-proliferative effect in HuCCA-1 cells, but GA (at $0.5-2.5 \mu \mathrm{M}$ ) failed to induce apoptosis and cell cycle arrest in HuCCA-1 cells (Figure $\mathrm{S} 1 \mathrm{~A})$ which raises an intriguing question of whether 
acquired resistant mechanism mediated by GA is a cell line dependence. However, further investigation of the molecular mechanisms govern HuCCA-1 resistance should be addressed.

Aberrant activation of $\mathrm{Wnt} / \beta$-catenin signaling cascade is implicated in a variety of tumors, including hepatocellular tumor (Nejak-Bowen and Monga, 2011) and CCA (Boulter et al., 2015). Unlike in HEK293 cells, we observed that the inhibition of $\beta$-catenin was not directly regulated by the phosphorylation of GSK-3 $\beta$ in CCA (Figure S2C). We thus anticipated the possibility that GA-inhibited $\beta$-catenin expression in this cell line may be regulated by other components of Wnt signaling pathway such as AXIN2 which is one of an important $\mathrm{Wnt} / \beta$-catenin target gene and acts as a negative regulator of $\mathrm{Wnt} / \beta$-catenin signaling through a negative feedback mechanism (Jho et al., 2002). The expression of AXIN2 was up-regulated during activation of $\mathrm{Wnt} / \beta$-catenin signaling to promote the differentiation of mouse pre-osteoblastic cells (Bhukhai et al., 2012), while in our current study, AXIN2 was significantly increased, despite a decrease in $\beta$-catenin protein expression by GA in KKU-M213 cells. The results corroborate well with the previous study showing that $A X I N 2$ has been demonstrated to promote degradation of $\beta$-catenin (Bernkopf et al., 2015). At present, the underlying mechanism is still unclear. However, elucidating the effect of GA on upstream components of Wnt signaling pathway which directly regulate the degradation of $\beta$-catenin should be considered in the future for a better understanding of the molecular mechanisms of GA.

CHOP is one of the key downstream molecules involved in ER stress-mediated apoptosis (Han et al., 2019). It should be noted that the XBP1 mRNA was significantly decreased by GA (Figure 3D) but there was a significantly increased $C H O P$ mRNA expression (Figure 3C). These data were consistent with a previous study showing that knockdown of XBP1 significantly up-regulated $C H O P$ protein leading to an increase in apoptosis rate and cell cycle arrests in mouse granulosa cells (Wang et al., 2017). Thus, we anticipated that the reduction of $X B P 1$ by GA may cooperate the result in the induction of apoptosis and cell cycle arrest through activating CHOP. In addition, GEM is a first-line therapy for pancreatic ductal adenocarcinoma and it has been explored as a single agent or in combination treatments (Gulhati et al., 2019). However, gemcitabine resistance appears to be a major obstacle of chemotherapy in CCA (Sawasdee et al., 2020). Here we showed that GA sensitized CCA cells to GEM (Figure 3F) and the combination of GA could be envisaged to overcome gemcitabine resistance in CAA patients.

In summary, our data demonstrates the significant biological activities of GA against CCA cell growth through the inhibition of $\mathrm{Wnt} / \beta$-catenin signaling and modulation of ER stress induced-apoptosis. GA holds promise as an anticancer and it may represent as a novel therapeutic option for CCA which could be exploited to minimize the use of gemcitabine in the setting of the combination treatment with the current chemotherapy.

\section{Author Contribution Statement}

K.B., K.S., K.J., and A.C. conceived and planned the experiments. K.S, K.J., S.R., and K.B. performed the experiments. K.B., K.S, K.J., N.A., V.R., N.T., and A.C contributed to analysis and interpretation of the results. K.B., K.S, K.J., N.T., and A.C wrote the original manuscript. All authors reviewed and approved the final manuscript

\section{Acknowledgements}

We thank Dr. Banchob Sripa from Liver Fluke and Cholangiocarcinoma Research Center, Department of Pathology, Faculty of Medicine, Khon Kaen University for providing KKU-M213 cells, Dr. Stitaya Sirisinha, from Department of Microbiology, Faculty of Science, Mahidol University for providing HuCCA-1 cells, and Dr. Pawinee Piyachaturawat from Department of Physiology, Faculty of Science, Mahidol University for critical reading and comments on the manuscript.

\section{Funding statement}

This project was supported by Mahidol University (Basic Research Fund: fiscal year 2021) and Faculty of Science, Mahidol University, Thailand Science Research and Innovation (Thailand Research Fund) (MRG62 to $\mathrm{KB}$ ), the research grants through the International Research Network (IRN58W0004) and partially supported by the Central Instrument Facility (CIF), Faculty of Science, Mahidol University.

\section{Ethical Approval}

The laboratory biosafety was approved from the Biosafety Committee of Faculty of Science, Mahidol University.

\section{Statement conflict of interest}

Authors have no conflict of interest to disclose.

\section{References}

Abdel-Rahman O, Elsayed Z, Elhalawani H (2018). Gemcitabinebased chemotherapy for advanced biliary tract carcinomas. Cochrane Database Syst Rev, 4, CD011746.

Alsaleh M, Leftley Z, Barbera TA, et al (2019). Cholangiocarcinoma: a guide for the nonspecialist. Int $J$ Gen Med, 12, 13-23.

Banales JM, Cardinale V, Carpino G, et al (2016). Expert consensus document: Cholangiocarcinoma: current knowledge and future perspectives consensus statement from the European Network for the Study of Cholangiocarcinoma (ENS-CCA). Nat Rev Gastroenterol Hepatol, 13, 261-80.

Bernkopf DB, Hadjihannas MV, Behrens J (2015). Negativefeedback regulation of the Wnt pathway by conductin/ axin2 involves insensitivity to upstream signalling. $J$ Cell Sci, 128, 33-9.

Bhukhai K, Suksen K, Bhummaphan N, et al (2012). A phytoestrogen diarylheptanoid mediates estrogen receptor/ Akt/glycogen synthase kinase 3 beta protein-dependent activation of the $\mathrm{Wnt} /$ beta-catenin signaling pathway. $J$ Biol Chem, 287, 36168-78.

Boulter L, Guest RV, Kendall TJ, et al (2015). WNT 
signaling drives cholangiocarcinoma growth and can be pharmacologically inhibited. J Clin Invest, 125, 1269-85.

Chairoungdua A, Smith DL, Pochard P, et al (2010). Exosome release of beta-catenin: a novel mechanism that antagonizes Wnt signaling. J Cell Biol, 190, 1079-91.

Chanchai C, Piyasatit P, Muntham D, et al (2019). Clinical prognostic factors and treatment outcomes for the survival of patients with cholangiocarcinoma in the Eastern Region of Thailand. Asian Pac J Cancer Care, 4, 101-5.

Chansitthichok S, Chamnan P, Sarkhampee P, et al (2020). Survival of patients with cholangiocarcinoma receiving surgical treatment in an O. viverrini Endemic Area in Thailand: A Retrospective Cohort Study. Asian Pac J Cancer Prev, 21, 903-9.

Gulhati P, Prakash L, Katz MHG, et al (2019). First-line gemcitabine and nab-paclitaxel chemotherapy for localized pancreatic ductal adenocarcinoma. Ann Surg Oncol, 26, 619-27.

Hahnvajanawong C, Boonyanugomol W, Nasomyon T, et al (2010). Apoptotic activity of caged xanthones from Garcinia hanburyi in cholangiocarcinoma cell lines. World J Gastroenterol, 16, 2235-43.

Han M, Gao H, Xie J, et al (2019). Hispidulin induces ER stress-mediated apoptosis in human hepatocellular carcinoma cells in vitro and in vivo by activating AMPK signaling pathway. Acta Pharmacol Sin, 40, 666-76.

He XY, Liu XJ, Chen X, et al (2013). Gambogic acid induces EGFR degradation and Akt/mTORC1 inhibition through AMPK dependent-LRIG1 upregulation in cultured U87 glioma cells. Biochem Biophys Res Commun, 435, 397-402.

Jho EH, Zhang T, Domon C, et al (2002). Wnt/beta-catenin/ Tcf signaling induces the transcription of Axin2, a negative regulator of the signaling pathway. Mol Cell Biol, 22, 1172-83.

Kashyap D, Mondal R, Tuli HS, et al (2016). Molecular targets of gambogic acid in cancer: recent trends and advancements. Tumour Biol, 37, 12915-25.

Marin JJG, Lozano E, Herraez E, et al (2018). Chemoresistance and chemosensitization in cholangiocarcinoma. Biochim Biophys Acta Mol Basis Dis, 1864, 1444-53.

Nejak-Bowen KN, Monga SP (2011). Beta-catenin signaling, liver regeneration and hepatocellular cancer: sorting the good from the bad. Semin Cancer Biol, 21, 44-58.

Nusse R, Clevers H (2017). Wnt/beta-Catenin signaling, disease, and emerging therapeutic modalities. Cell, 169, 985-99.

Prakobwong S, Suwannatrai A, Sancomerang A, et al (2017). A large scale study of the epidemiology and risk factors for the carcinogenic liver fluke opisthorchis viverrini in Udon Thani Province, Thailand. Asian Pac J Cancer Prev, 18, 2853-60.

Qin Y, Meng L, Hu C, et al (2007). Gambogic acid inhibits the catalytic activity of human topoisomerase IIalpha by binding to its ATPase domain. Mol Cancer Ther, 6, 2429-40.

Reutrakul V, Anantachoke N, Pohmakotr M, et al (2007). Cytotoxic and anti-HIV-1 caged xanthones from the resin and fruits of Garcinia hanburyi. Planta Med, 73, 33-40.

Sawasdee N, Thepmalee C, Sujjitjoon J, et al (2020). Gemcitabine enhances cytotoxic activity of effector T-lymphocytes against chemo-resistant cholangiocarcinoma cells. Int Immunopharmacol, 78, 106006.

Tripathy A, Thakurela S, Sahu MK, et al (2018). The molecular connection of histopathological heterogeneity in hepatocellular carcinoma: A role of Wnt and Hedgehog signaling pathways. PLoS One, 13, e0208194.

Vaeteewoottacharn K, Kariya R, Matsuda K, et al (2013). Perturbation of proteasome function by bortezomib leading to ER stress-induced apoptotic cell death in cholangiocarcinoma. J Cancer Res Clin Oncol, 139,
1551-62.

Verras M, Papandreou I, Lim AL, et al (2008). Tumor hypoxia blocks Wnt processing and secretion through the induction of endoplasmic reticulum stress. Mol Cell Biol, 28, 7212-24.

Vogel A, Wege H, Caca K, et al (2014). The diagnosis and treatment of cholangiocarcinoma. Dtsch Arztebl Int, 111, 748-54.

Wang N, Zhao F, Lin P, et al (2017). Knockdown of XBP1 by RNAi in mouse Granulosa cells promotes apoptosis, inhibits cell cycle, and decreases estradiol synthesis. Int $J$ Mol Sci, 18.

Wang W, Zhong W, Yuan J, et al (2015). Involvement of Wnt/beta-catenin signaling in the mesenchymal stem cells promote metastatic growth and chemoresistance of cholangiocarcinoma. Oncotarget, 6, 42276-89.

Wu X, Deng G, Hao X, et al (2014). A caspase-dependent pathway is involved in Wnt/beta-catenin signaling promoted apoptosis in Bacillus Calmette-Guerin infected RAW264.7 macrophages. Int J Mol Sci, 15, 5045-62.

Yao D, Kunam VK, Li X (2014). A review of the clinical diagnosis and therapy of cholangiocarcinoma. $J$ Int Med Res, 42, 3-16.

Zhang HZ, Kasibhatla S, Wang Y, et al (2004). Discovery, characterization and SAR of gambogic acid as a potent apoptosis inducer by a HTS assay. Bioorg Med Chem, 12, 309-17.

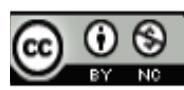

This work is licensed under a Creative Commons AttributionNon Commercial 4.0 International License. 